\title{
Interested in Neurosurgery as a Career
}

Last Updated: March 16, 2020

\section{Why Neurosurgery?}

"You can't ever reach perfection, but you can believe in an asymptote toward which you are ceaselessly striving." - from When Breath Becomes Air, by Paul Kalanithi, MD, late Stanford neurosurgery resident

Most neurosurgeons can probably identify what initially attracted them to their specialty: a specific patient's story, a compelling research project, a love of the operating room, or an inspirational mentor. Ask a neurosurgeon what his or her reasons for choosing neurosurgery were; regardless of the proximate causes, several common themes seem to draw most neurosurgeons to this career.

- Relationship with Patients: The bond between neurosurgeon and patient is unique in all of medicine. Neurosurgeons operate on the brain and the spine, which are unlike any other organ in that they house our movements, speech, thoughts, and personalities. The level of trust that develops between neurosurgeons and their patients is unmatched, and neurosurgeons develop long-lasting relationships with their patients as a result.

- Patients Themselves: it goes without saying that the 
patients who require neurosurgical intervention are some of the sickest in the hospital. Many endure unspeakable suffering before seeking care, many do not fully recover, and many pass away. Neurosurgeons consider it an honor to take care of these patients and shepherd them and their families through what is often the most difficult part of their lives.

- Work Ethic: neurosurgeons and neurosurgery residents are often the first doctors in the hospital and are often the last to leave. They have a singular focus on achieving the best possible outcomes for their patients. Indifferent to the time of day or night, neurosurgeons are always ready to go above and beyond in order to help their patients.

- Pursuit of Excellence: The pursuit of excellence has been an inherent aspect of neurosurgery since its inception at the hands of legendary figures such as Harvey Cushing, Walter Dandy, and Gazi Yasargil. There is an ideal that neurosurgeons, by virtue of the organs they operate on, are driven to strive for perfection. It is intrinsically inspirational to surround oneself with people who are not satisfied to meddle in mediocrity but are constantly pushing themselves towards excellence.

- Research: Paul Kalanithi also wrote, "the ethos of neurosurgery - of excellence in all things - maintains that excellence in neurosurgery is not enough." In this spirit, neurosurgery is a field that is very closely integrated with neuroscience research. Elective time for 
research is built into almost every residency program, and most academic neurosurgeons participate in some type of research, from basic neurobiology, to clinical research, and every neuroscience-related topic in between. Neuroscience is a rapidly expanding scientific discipline still in its infancy, and the unique access that neurosurgeons have to the human brain and spine is an extremely valuable asset to the field.

- Colleagues: neurosurgeons are among the most dedicated, driven, and passionate people in the hospital, and it is easy to find an inspirational senior colleague as a mentor. Neurosurgery is a small, but very tight-knit, elite community; residents and attendings run into each other regularly at national conferences, and many are close friends outside the professional setting, having met first on the residency interview trail. Mostly gone are the days of malignant hospital environments; most resident cohorts consider each other family.

- Anatomy: Cerebral and spinal anatomy is mesmerizing; it is beautiful, intricate, and most importantly the crown jewel of creation. Like the Everest summit that needs to be conquered, many neurosurgeons enter neurosurgery with the desire to master and understand the complex neurosurgical anatomy.

- Microneurosurgery: The technical skills required provide a stimulating endeavor that is rewarded by excellent outcomes. Taking on this technical challenge provides a unique opportunity for the surgeon to differentiate 
himself or herself from other members of the surgical team.

\section{Understanding Neurosurgery as a Career Path}

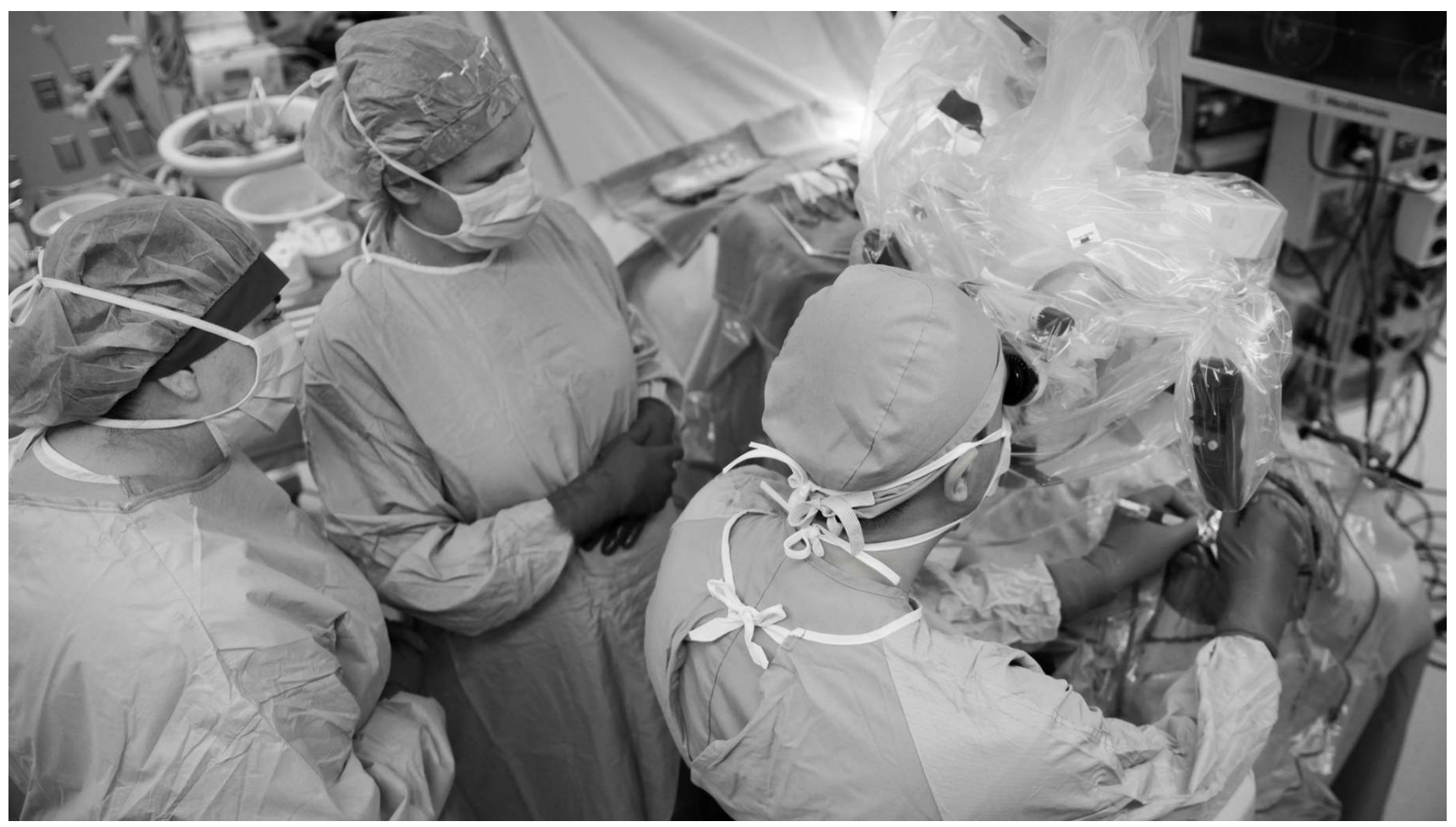

The neurosurgical training path or trajectory in the United

States generally consists of the steps outlined below:

- Medical school: 4+ years in an accredited MD or DO program (or foreign equivalent). Most medical students interested in neurosurgery will pursue significant research in medical school, potentially including a combined MD/PhD degree. Near the end of the first clinical year or during the second clinical year, the majority of students complete subinternships (sub-Is) in neurosurgery at their home institution and/or a number of other institutions in preparation to apply for residency through the National Resident Matching Program (NRMP). 
- Residency: Seven years in an ACGME-accredited residency program, with each program accepting 1-4 residents each year. Program structure varies greatly, but generally follows a basic outline:

- PGY1 (internship): various rotations in neurosurgery, neurology, neuropathology, neuroradiology, neurosciences ICU, or other surgical specialties

- PGY2-3 (junior residency): primary resident taking in-house call, admitting new patients to the service, rotating through the neurosurgical services, while learning basic procedures in the operating room

- PGY4-6 (senior residency): continuing to rotate through different neurosurgical services and developing surgical skills associated with increasingly more complex operations. Of note, 1-2 years of elective, protected time for research or enfolded clinical fellowships are usually built into these years.

- PGY7 (chief residency): in charge of the neurosurgical service and educating junior residents. Operating daily on the most complex cases on service and honing the skills specific to the resident's chosen subspecialty.

- Fellowship: For 1-2 years after residency. A fellowship is required for further specialization in pediatric neurosurgery, and is optional but often undertaken to develop the skills necessary for an academic position in 
subspecialties such as cerebrovascular and endovascular neurosurgery, complex and minimally invasive spine surgery, skull base surgery, and functional neurosurgery. Many residency programs offer "enfolded" fellowships, where the 1-2 years of protected elective time may be used to focus on the resident's subspecialty of choice, thereby obviating the need for extra training after residency. The rules regarding accreditation for fellowships are currently in flux; expect them to be different by the time you are a resident. These rules will be updated in this guide.

- Career: Clinical neurosurgeons generally follow one of two paths: academic or private practice. Most academic neurosurgeons work at university-affiliated medical centers and are responsible for seeing patients in clinic, operating, and teaching residents, fellows, and medical students.

Academics involve active research participation, either as the principal investigator of a laboratory or as a collaborator, in either bench-based research, clinical research, or engineering/innovations work. Private practice neurosurgeons often work for small to mid-sized group practices and have operating privileges at one or several hospitals, where they see their own patients. There are other career options available to neurosurgeons, of course, in the biotechnology, pharmaceutical, and medical device industries, as well as 
in finance, military or government services.

\section{Advantages and Disadvantages of a Career in Neurosurgery}

As with any career choice, neurosurgery has advantages and disadvantages:

- Advantages:

- The chance to help the sickest patients in the hospital with devastating neurological diseases

- Technically challenging operations on fascinating and complex anatomy

- Motivated and passionate colleagues

○ Endless research opportunities

- Disadvantages:

- Extensive training path

- Long hours, call, and schedule are often inflexible

- Potential personal sacrifices in terms of relationships, family, and hobbies

- Emotional discomfort of witnessing incredibly sick patients suffer

\section{I am interested in neurosurgery, but everyone keeps telling} me not to do it!

Medical students interested in neurosurgery may be told by friends, family, peers, physicians, and even neurosurgeons, that they should pursue another field. As with all pieces of advice, be sure to assess the context and the intentions of 
whoever gave them, and always take the advice with a grain of salt.

In general, advice from someone who is not in the medical field regarding which specialty you should choose can be taken less seriously than, for instance, a surgeon who has sacrificed a great deal and provides the advice from hopefully relatively objective experience. The best advice is the one that most accurately applies to your life circumstances (though this may not always be obvious at the time). Take your individual situation into account and remember that others' experiences, while valuable, will almost always be different from your own.

If advised not to pursue neurosurgery, it is important to ask yourself, and even the adviser, why he or she made that suggestion. Is the adviser asking you to be more honest with yourself and to reflect on your personality - which may be acutely affected by stress and patients' outcomes? Is it a family friend, who is a physician, telling you what a "horrible" lifestyle neurosurgery involves? Sometimes, advice discouraging you from pursuing your goals to the fullest can be a reflection of the advisor's own limitations. Therefore, although it is valuable to ask around and get as much advice as you can, please consider the context of the advice and the perspective of the giver.

Neurosurgeons providing the "don't do it" advice often do so with a specific goal in mind. First, they may do this to ensure that you are committed. If you still want to become a 
neurosurgeon, even after a neurosurgeon tells you not to, this can demonstrate unyielding strength of character, a quality that will serve you well in life. Second, neurosurgeons may feel it is their responsibility to ensure that you thoroughly explore other fields before deciding on neurosurgery.

Neurosurgical applicants often say that they do not think they would be happy doing anything else. This is an extreme statement, yet it may be true for you. Others have several interests, but possess the drive and passion to succeed in any of them. In either case, you would be doing yourself a great disservice by pursuing the grueling training of neurosurgery without taking the time to solicit advice and make a wellinformed decision.

Lastly, a neurosurgeon may try to sway you away from neurosurgery because he or she is unhappy with his or own decision.

A full discussion of all the reasons a practic might feel this way is beyond the scope suffice it to say that shifting norms arg autonomy and compensation have in surgical practice over the years $t$ disappointing to some. In any eve ask why this particular advice wa

As noted above, great sacrifices a e pursuit of neurosurgery. One of the greates le limitations that neurosurgery imposes on time-spent with 
friends and family, especially during residency. As a result, women and men who know that having a large family is important to them, will often opt out of specialties with rigorous training programs.

The fact that women often bear the brunt of childcare responsibilities, however, provides an extra dimension for them to think about when considering a neurosurgical career. The issues of pregnancy, birth, and childcare, compounded with the fact that only $10 \%$ of board-certified neurosurgeons in the US are female, means that the recipients of advice discouraging students from pursuing neurosurgery are often women.

For the women (and men) who have received this "don't do it" advice, we offer our thoughts to complement everything else you have been told:

- Be thankful to the hundreds of women who have pursued this path before you - they have done much of the heavy lifting. They are incredible trailblazers and have accomplished a great deal. To read more about these women and the history of women in neurosurgery, visit Women in Neurosurgery (WINS).

- The future of neurosurgery is dependent on women entering the field. In 2008, the AANS released a statement saying that "fairness and equality are fundamentally right and vital to the future success of our evolving specialty". That same year, WINS released a 
white paper about the recruitment and retention of women in neurosurgery. These pieces outline the hurdles to including more women in neurosurgery, and more importantly, ways to overcome them.

- For women interested in neurosurgery, finding female neurosurgical mentors can be valuable. Although this is not absolutely necessary, as many successful female neurosurgeons have had excellent male mentors, it can be helpful to obtain a different perspective. Use national conferences as opportunities to meet, connect, and learn from these accomplished women. We suggest beginning with the AANS/CNS joint group, Women in Neurosurgery (WINS).

- Planning children around residency training is difficult for men and women alike. Some residents opt to wait until training is complete so that a more flexible schedule exists, though the length of neurosurgical training often runs up against biological constraints. Others have had children during residency, often with friends and family members nearby to help. Some residents choose to hire help at a considerable cost. Applicants should remember that nothing is absolute or impossible, and having children is always easier with the right support system. In the end, make decisions that are best for you, your family and your career.

- Remember that it is inappropriate for interviewers to ask about your relationship status or your plans for becoming pregnant or having children. However, many 
applicants in recent cycles report that these questions are still fairly common. We recommend consulting with a trusted advisor for guidance on how to navigate this difficult situation.

\section{We hope that readers of all genders follow their dreams and} remain true to themselves, regardless of which specialty they choose.

\section{I am interested in neurosurgery - who do I contact?}

The answer depends on whether or not your medical school owns a neurosurgery residency program. If you are a medical student at a medical school with a neurosurgery residency program, the best place to start would be to contact the chairman of the department and/or neurosurgical residency program director for a short introductory meeting. Either before or after this meeting, getting connected with residents at the program is incredibly useful, as they will be one of the best sources of research projects and mentorship regarding the application process.

If you are a medical student at a school that does not have a residency program, reach out to any neurosurgery faculty and/or peer who is pursuing neurosurgery. Reaching out to a private practice neurosurgeon who is contracted into a hospital affiliated with your medical school is also an acceptable place to start, but it is preferable that you 
the match process. In addition, private practice neurosurgeons are less likely to offer research projects.

Neurosurgery is a very small field and acts in many ways like a "family". This is especially true for academic neurosurgery. It is safe to assume that most academic neurosurgeons know each other, at least in passing. Thus, it is important to become acquainted with academic neurosurgeons at your institution as they will often provide introductions and additional communication to neurosurgical faculty at other residency programs.

Additionally, neurosurgical faculty are able to provide a framework/timeline for the tasks and goals that will help ensure a successful match process. In general, every applicant should at least consider a future academic neurosurgery career and attempt to provide a lasting contribution to the field, as the majority of candidates will identify this as a goal during the interview process, and interviewers are primarily looking for residents who will contribute positively to their institution's research efforts. 


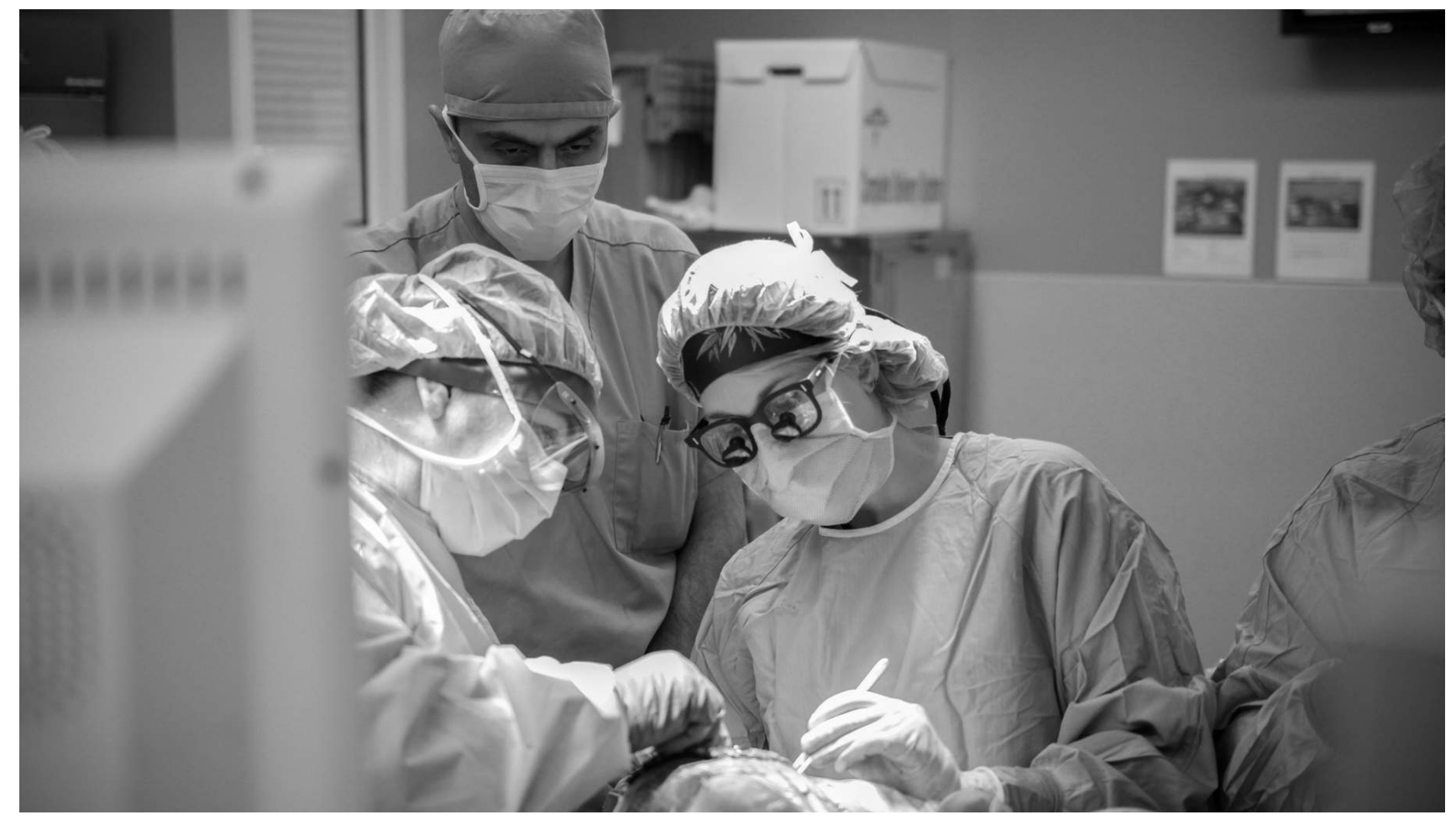

The influence of a neurosurgical mentor on your medical school career, residency match, and ultimately your career trajectory cannot be overstated. A strong mentor will be able to provide guidance throughout medical school and will ultimately strengthen the residency application.

In general, these mentors will get to know you the best and ultimately write your letters of recommendation. As such, it is essential to seek out mentors early, even as early as the first year of medical school. This will ensure that you receive adequate guidance while developing a strong relationship which will be reflected in your letters of recommendation.

When selecting a mentor, it is preferable to speak with medical students who are currently in the application process or have recently matched into neurosurgery from your institution. They will be great resources - not only about the 
have a strong interest in mentoring medical students.

Once you have selected a neurosurgical mentor, it is important to demonstrate your work ethic. While it is necessary to tailor your approach to the specific mentor you have selected, remember that your mentor is busy. Key tips for working with a neurosurgical mentor are: 1) Set clear expectations and then work harder than your mentor expects you to. If he or she gives you 4 weeks to complete a literature review, email them an organized literature review in 2 weeks. If they ask you to try to get through the first 100 chart reviews before your next meeting, finish the next 100 as well. Begin building a reputation of someone who goes above and beyond. 2) Your research mentor should never have to ask for updates from you. Send monthly updates about all of the projects you have undertaken with your mentor and the progress you've made over the previous month. 3) A mentor's responsibility is to promote his or her mentee but it is also a mentee's responsibility to promote his or her mentor. Praise them to other faculty members and let them know the efforts your mentor has shown in helping and teaching you. Always remember that you are where you are as a result of their hard work and effort on your behalf. Ultimately, as you develop this strong relationship, he or she will trust you with more projects, more responsibilities in the $\mathrm{OR}$, and he or she will be more comfortable introducing you to their influential 
neurosurgical mentor will be created by means of working together on various projects. Most faculty enjoy this working relationship since it is based on honest enthusiasm on both sides.

\section{How to Communicate with Your Mentor}

Once you have decided who you would like to work with, plan on writing an email to introduce yourself, your particular interests, and what type of skills or experience you have to offer. Try your best to understand what kind of work your potential mentor works on and briefly describe why it excites you. More importantly, try to explain how interested you are to get involved and why. Your updated CV should be attached and referenced in the email. Do your best to write professionally and in a succinct manner. Ideally, a resident you know well will advocate for you and follow up your email with one of his or her own. An example of such an email is as follows:

\section{Dear Dr. X,}

My name is [first and last name] and I am a [year in medical school] student at [school]. I am interested in pursuing a career in neurosurgery and your work in [field or study] interests me tremendously. I have previously worked with [x scientist/doctor/researcher] on [x] and my skill set includes [ $\mathrm{x}$ and $\mathrm{x}$. If you have an opportunity for me, I am interested in contributing to [specific project] or any other project you would have in mind. My schedule during the coming 
[summer/year] is amenable to being involved in a research project [part/full] time (note: neurosurgeons don't really believe in part-time anything!). My CV is attached. Please let me know if you would require any other information. I look forward to hearing from you and hopefully meeting you in person.

Sincerely,

Name

This example is very basic, and you should cater it to the type of person to which you are writing. If you do not have any previous research experience, that is also acceptable. You are free to mention any other experience or skill you feel would contribute to your productivity or just mention how eager you are to get involved. Also, do not be afraid of rejection. If someone does not have time to help you, thank them and look for someone else to contact.

Sometimes, it can be more efficient to connect with a resident or fellow who is currently working with a faculty in your department. Either the faculty member will connect you with them, or you can contact them directly. The residents and fellows are generally the most approachable; they will directly report to the corresponding faculty about your work. It would be a great idea to meet residents in person, possibly shadowing them early during medical school to get a feel for the specialty. This will also give you the ideal platform to ask to get involved in research and possibly find out which faculty are most open and willing to take a student on. 
Once you have connected with a resident and/or neurosurgeon, it is important to remember how busy they are. When communicating with them, keep emails brief and to a minimum in number; always be succinct and efficient.

\section{Grant and Funding Opportunities}

Some students are able to successfully balance demanding laboratory research projects with the medical school curriculum; however, this ability depends on your time management skills and the opportunities available in your lab.

Many funding opportunities for medical student research represent a wonderful chance to practice grant writing skills. Obtaining one of these awards is a tremendous experience and bolsters the competitiveness of your application. Some examples of these awards, and much more information about pursuing research in medical school, can be found in Section titled Conducting and Presenting Research.

\section{DOI:}




\section{Submit Your \\ Complex Case}

to be reviewed by

the Atlas team. 


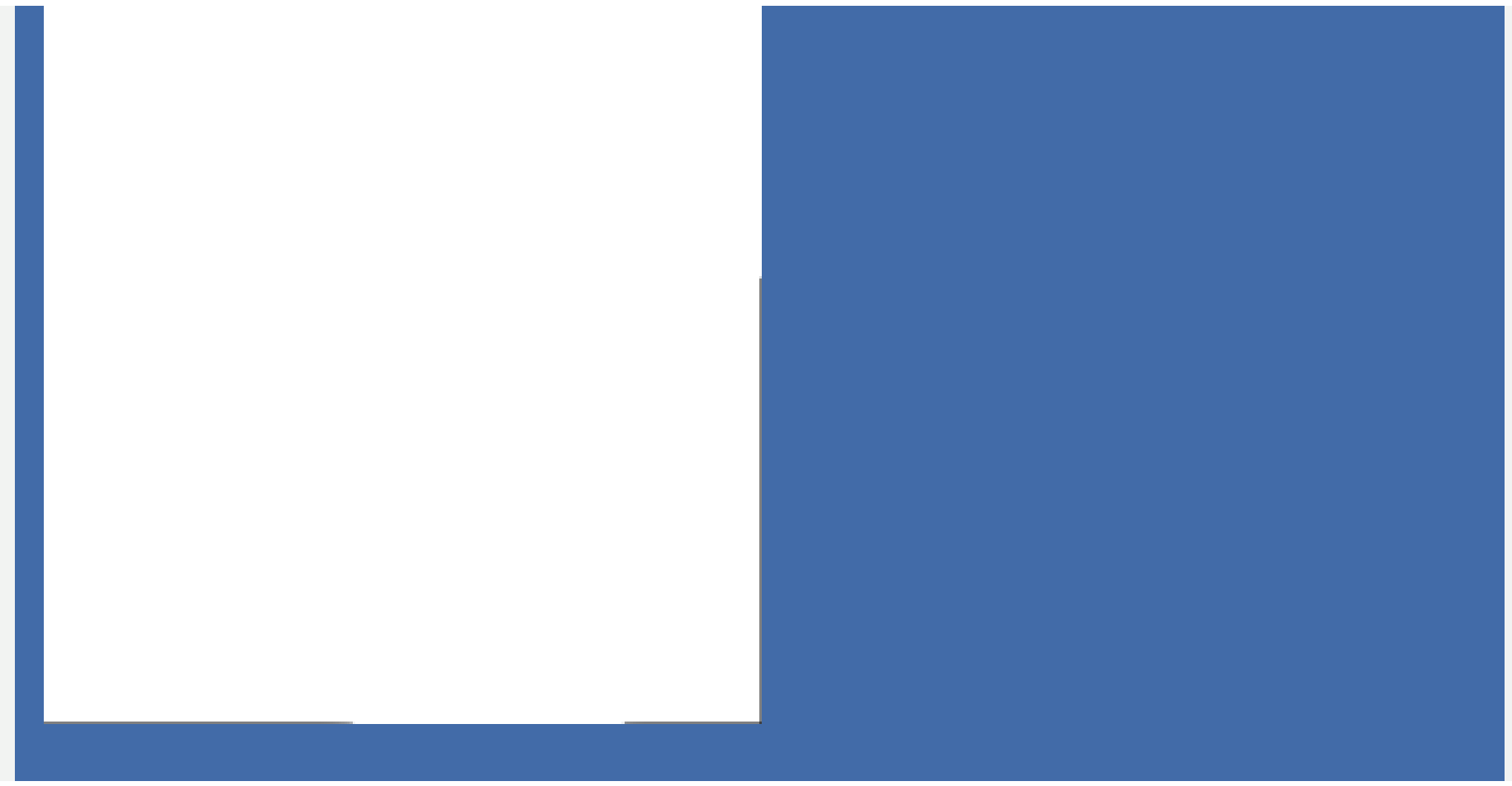

